\title{
EDUCACIÓN VIRTUAL: OPCIÓN DE ESTUDIOS PARA LOS BACHILLERES EN LA CIUDAD DE GUAYAQUIL
}

Carlos Eduardo Mora Torres, MBA.

Máster en Administración de Empresas (España). Supervisor de auditoría en la Compañía RGPACCIOLI\&COMPANY S.A., Ecuador. cmora@rg-paccioli.com

Franklin Duberlí Castillo Llanos, CPA.

Contador Público autorizado (Ecuador). Labora en la Compañía CEPROFISH S.A., Ecuador. contabilidad@ceprofish.com

Génesis Karina Plaza Alarcón, CPA.

Contador Público autorizado (Ecuador). Labora en la Compañía WINEDTECH S.A., Ecuador. asistentecontable@winedtech.com

\section{ARTÍCULO DE INVESTIGACIÓN}

Recibido: 8 de agosto de 2019.

Aceptado: 6 de noviembre de 2019.

\section{RESUMEN}

Este artículo tiene por objeto analizar el grado de aceptación de la educación virtual que tienen los estudiantes del último año de Educación Básica Superior de 5 colegios de la zona norte y sur de la ciudad de Guayaquil pertenecientes a los distritos que presentan mayores tasas de estudiantes que no logran obtener un cupo universitario en una Institución de Educación Superior. El estudio se sustentó en los postulados teóricos de Chen, Sorlin y Vessuri, Silva, López Tovar, entre otros. El trabajo se enmarcó como no experimental de campo, transversal y descriptivo. La población de estudio se conformó por un grupo de 150 estudiantes sin discriminar entre géneros ni rendimiento estudiantil. Los resultados mostraron que los estudiantes consideran estar de acuerdo con seguir sus estudios de manera virtual, de no alcanzar cupo para la Institución de Educación Superior que habían decidido aplicar para sus estudios presenciales.

Palabras clave: aula virtual, nuevas tecnologías, formación profesional, modelo pedagógico, 
campus virtual.

\section{ABSTRACT}

The aim of this article is to analyze the degree of acceptance of the virtual education that the students of the last year of Higher Basic Education have of 5 schools of the north and south zone of the city of Guayaquil belonging to the districts that present higher rates of students than they do not manage to obtain a university quota in a Higher Education Institution. The research is based on the theoretical postulates of Chen, Sorlin and Vessuri, Silva, López Tovar, among others. The study was framed as non-experimental field, cross-sectional and descriptive. The study population was formed by a group of 150 students without discriminating between genres or student performance. The results showed that the students consider to be in agreement with continuing their studies in a virtual way, of not reaching a quota for the Institution of Higher Education that they had decided to apply for their face-to-face studies.

Keywords: virtual classroom, new technologies, professional training, pedagogical model, virtual campus.

\section{INTRODUCCIÓN}

Las condiciones sociales, políticas, económicas y culturales que caracterizan a las sociedades del siglo XXI han permitido, entre otras cosas, el surgimiento de lo que se conoce como la cultura de la sociedad digital (Lévy, 2007), en la cual se hayan inmersas principalmente las nuevas generaciones de alumnos, puesto que utilizan las tecnologías digitales como las formas dominantes para comunicarse, compartir información y conocimiento, investigar, producir, organizarse y administrar.

En Ecuador, se considera que la Educación Virtual es nueva e innovadora, por ello se manifiesta como una fuerte competencia en el esquema tradicional de la educación; que se distingue principalmente por ser un sistema presencial convencional en el que el docente y el alumno comparten un espacio físico común donde se imparte el aprendizaje. Herman (2009) puntualiza que utilizar la educación virtual en las aulas de clases no solo se debe ver como innovación tecnológica, sino más bien, como una estrategia que contribuye a mejorar el proceso de enseñanza-aprendizaje.

En Educación, es necesario utilizar la tecnología con todos sus avances y de la manera más eficaz. Para Sorlin \& Vessuri (2007) se trata del siglo de las universidades, esto por ser un proceso innovador que coincide con la inclusión generalizada de jóvenes a los nuevos 
sistemas de enseñanza. Uno de los grandes retos que tiene por delante la educación del siglo XXI en Ecuador, es mantenerse al corriente con las Nuevas Tecnologías de la Información y la Comunicación que permiten una nueva forma de expresión. "La clave reside en establecer su sentido y aportación en el proceso de enseñanza-aprendizaje" (Silva, 2009, p. 25).

En los tiempos actuales la educación superior en América Latina, especialmente en Ecuador, está incorporando nuevas formas de gestionar el trabajo educativo realizando parte o la totalidad de su actividad en formato virtual, esto ha llevado a que se aprueben marcos legales que autorizan a las universidades el poder ofertar programas académicos virtuales en países como Ecuador, Colombia, México, Costa Rica, Argentina, El Salvador, Paraguay, Panamá, y Puerto Rico (Martínez y Romero, 2017). Además, instituciones de educación superior públicas tradicionalmente presenciales en el marco de sus reglamentos, han ido incorporando ofertas académicas únicamente virtuales, así como otras que se han especializado en la oferta virtual casi en su totalidad con una sola excepción la rendición de exámenes que siguen siendo de manera presencial (Gazzola y Didriksson, 2016, p. 62).

A su vez, Unigarro (2004) recalca que para algunas personas es un error expresarse como educación virtual, pues dice, la educación es un acontecimiento real, siendo un mejor término definirlo como educación en línea, educación con medios virtuales, entre otros.

La educación presencial tiene como metodología regular la clase magistral impartida dentro del aula de clase, la cual se centra en la capacidad pedagógica, conocimientos y experiencia del docente, así como la asistencia física de los alumnos. Por otro lado, la educación virtual se apoya en la diversidad de recursos que facilita la tecnología como Internet, plataformas digitales, buscadores de información, etc. (García, 2017).

El correcto manejo de la educación virtual en el mundo ha logrado que la educación presencial no sea la única alternativa para que los estudiantes inicien su carrera universitaria. Este potencial educativo permite a los alumnos, mediante la metodología virtual, asistir a cursos planificados conforme a su disponibilidad de tiempo y ajustado a su ritmo de aprendizaje, optimizando por otra parte el aporte de los profesores que en su nuevo rol de "tutores", preparan el material educativo, asisten, guían y evalúan a los cursantes para garantizar la calidad del aprendizaje. Por tal razón, existen diferencias que un estudiante debe entender entre estas dos metodologías de estudio, las cuales se encuentran detalladas en la tabla 1.

Tabla 1. Diferencias entre educación presencial y educación virtual.

Presencial Virtual




\begin{tabular}{ll}
\hline $\begin{array}{l}\text { Existe un mayor vínculo entre el maestro y } \\
\text { el alumno, con lo cual la comunicación es } \\
\text { más directa y efectiva permitiendo el }\end{array}$ & $\begin{array}{l}\text { El estudiante es el centro del proceso } \\
\text { de aprendizaje y es un receptor activo } \\
\text { de la información entregada por los }\end{array}$ \\
\hline $\begin{array}{l}\text { El instructor transmite conocimiento y hay } \\
\text { menos probabilidades de obtener fallas }\end{array}$ & El ritmo y la manera de aprendizaje la \\
técnicas, por ejemplo, en materiales de & estar ligado al ritmo de la universidad \\
video y audio. & o institución a la que pertenezcas. \\
\hline $\begin{array}{l}\text { El horario está establecido y hay que } \\
\text { respetarlo para que se produzca el }\end{array}$ & $\begin{array}{l}\text { El constante uso de las TIC, potencian } \\
\text { el buen manejo de las herramientas }\end{array}$ \\
conocimiento. & tecnológicas. \\
\hline Los traslados a los centros educativos & Se fortalece los métodos de \\
hacen que la inversión en este modelo & comunicación. Tanto la comunicación \\
educativo aumente. & sincrónica, como la comunicación \\
\hline $\begin{array}{l}\text { Se mantendrán los métodos de enseñanza } \\
\text { comunes y el uso de las TIC será menor }\end{array}$ & Puede tomar las clases y consultar los \\
durante el proceso educativo, depende de & materiales educativos en cualquier \\
la creatividad del instructor mantener la & con conexión a internet. \\
atención de los estudiantes. &
\end{tabular}

Fuente: Elaboración propia.

El presente artículo tiene por objeto conocer el grado de aceptación de la educación virtual por parte de los estudiantes del último año de Educación Básica Superior de 5 colegios de la zona norte y sur de la ciudad de Guayaquil, próximos aspirantes al sistema universitario.

\section{REVISIÓN TEÓRICA}

Chen, et al. (2004) señala "que la enseñanza virtual se realiza en espacios donde los usuarios aplican un conjunto de estrategias de intercambio de información, basadas en sistemas de ordenadores, de redes telemáticas y de aplicaciones informáticas" (p. 59). Es por eso que se viene produciendo una transformación digital, individual y social que ha logrado poner a la educación en el lugar que le corresponde, como máximo responsable del avance de nuevas metodologías que ayuden a la evolución y crecimiento de la sociedad. Gracias a estas transformaciones, hoy en día se encuentra en auge todo lo referente a enseñanza online, con el objetivo de desarrollar procesos que ayuden al crecimiento profesional de los estudiantes que necesitan moverse a su propio ritmo. 
Según Álvarez, Rodríguez, Sanz y Fernández (2008) es necesario que los estudiantes se vuelvan aprendices autónomos, es decir, que busquen no solo los contenidos sino también el aprendizaje. Varias instituciones encargadas de la educación superior desde la modalidad de educación virtual buscan desarrollar módulos de calidad que ayuden a aumentar la posibilidad de alcanzar las máximas capacidades de los estudiantes para interactuar y relacionarse con sus tutores-docentes; aprendiendo en un entorno que se encuentra contenido por las tecnologías de la información y educación.

La evolución constante de la tecnología; así como la importancia que esta tiene actualmente sobre la educación, constituye un motivo que impulsa a las instituciones a pensar en el futuro de la enseñanza que logre llenar las expectativas de los estudiantes que buscan mejorar sus conocimientos y habilidades. Además, la educación virtual va en constante crecimiento día a día debido a sus ventajas, entre las cuales se encuentran el buen uso de la tecnología; la disminución de costos; sus aportes a la inclusión y acceso a la educación superior que en muchas ocasiones no logra abastecer a la gran demanda de estudiantes que quieren ingresar a las universidades (Noguera y Gros, 2016).

El desafío más grande que tienen en Ecuador y América Latina, en general, es romper la estigmatización que existe sobre la creencia que el modelo de educación virtual es sinónimo de mala calidad, lo cual no corresponde a la realidad actual, donde los avances tecnológicos, las herramientas y plataformas que se han desarrollado para el uso de la educación virtual, han logrado que alrededor del mundo entero las ofertas de programas a nivel de posgrado internacionales sean reconocidos por su gran calidad, así como por sus innovadores métodos pedagógicos (Rama, Domínguez y Rodríguez, 2016). Esto ha permitido que los procesos de formación permitan a los estudiantes, desarrollar múltiples competencias para entender los diferentes grados de complejidad que conlleva seguir el proceso académico con la misma o con mayor rigurosidad de los programas presenciales y de educación tradicional. Guido Eduardo Ochoa señala la falta de una adecuada aplicación de las facilidades de la educación virtual como medio de educación en el Ecuador (Ochoa, 2009).

López (2016) señala "El aprendizaje autónomo para poder sacar un mejor provecho, depende de otros factores que deben ser incluidos para reforzar las habilidades y actitudes de los estudiantes, como la personalidad de los tutores, quienes pasan de ser transmisores de conocimiento a facilitadores del aprendizaje" (p. 214). En los cuales se busca promover y orientar fundamentalmente la comunicación del grupo, motivación y creación de un clima atractivo para un mejor entendimiento; así como nuevas experiencias y sobre todo la construcción del conocimiento que logrará con el objetivo de obtener resultados educativos significativos, tanto para el aprendiz como para el propio tutor. 
Un componente importante en la evolución de la educación es que cada vez se van generando espacios más amplios y complejos. García y Seoane (2015) describen la evolución del elearning a partir de tres generaciones. La primera, llega caracterizada por el surgimiento de plataformas en línea que permiten un mejor aprendizaje; así como la generación de aulas y campus virtuales, las cuales tienen una continua evolución. La segunda, hace referencia al factor humano que permite la interacción como un elemento esencial entre compañeros del propio entorno o de diferente situación geográfica; y a su vez, profundiza la comunicación entre tutores y estudiantes para un e-learning de calidad que pretende ir más allá de un simple proceso de publicación de contenidos, para ser un enlace hacia un conocimiento más profundo. La tercera, se define porque el e-learning deja de estar relacionado de forma exclusiva a las plataformas de aprendizaje online y se transforma en un ecosistema tecnológico orientado hacia el proceso de aprendizaje.

Matallana y Torres (2011) manifiestan que la educación a distancia es cada vez más importante en cuanto a modelos educativos, ya que origina que las instituciones de educación superior se conviertan en instituciones generadoras de conocimiento, debido a que acogen a todos los estudiantes y permiten continuar su formación sin tener que cambiar su vida laboral, social y familiar. En este contexto, se asegura que actualmente tiene una mayor importancia y validez las modalidades a distancia y virtual al convertirse en posibilidades concretas frente a los retos y limitaciones relacionadas con calidad, cobertura, inclusión y factores económicos que afectan la acogida de personas en las modalidades presenciales dentro de los centros de educación superior. Tichapondwa \& Tau (2009) afirman que este nuevo modelo de educación también se constituye en una ventaja, debido a que el enfoque didáctico con que se realizan y diseñan los materiales a ser utilizados, ayudan al estudiante para que aprenda sin necesidad de tener de manera presencial al docente; el estudio es guiado, pero el estudiante cuenta con la suficiente libertad para tener flexibilidad en el manejo de su tiempo. Otra ventaja muy importante radica en que los adultos ya no necesitan escoger entre su trabajo o la culminación de estudios, puesto que esta modalidad de educación les permite ejercer de manera combinada ambas actividades.

Las universidades ven a futuro a la educación virtual como una forma más amplia para sus planes académicos, donde el objetivo es lograr un equilibrio con los estudios presenciales. Por otra parte, la educación virtual en América Latina sigue fortaleciéndose; cursos, especializaciones y maestrías están entre los programas que más acogida tienen, en donde con más experiencia bajo esta modalidad se encuentran Brasil y Argentina (Dávila y Ruiz, 2016, p. 14). 
Todavía en Ecuador quedan sectores relacionados con la educación que son cautos a la hora de incluir metodologías virtuales no solo por la inversión que llevaría realizar las plataformas que soporten este nuevo modelo de enseñanza, sino por el desconocimiento que tienen muchas personas sobre este tipo de enseñanzas únicamente virtuales, es por ello que en los últimos años se está comenzando a promoverlas con mayor fuerza en distintos países para así poder cerrar la brecha existente en la actualidad por la falta de cupos en las universidades.

En un estudio realizado por el World Economic Forum (2016) como se muestra en la figura 1, alrededor del $48 \%$ de las entrevistados afirmaron que están dispuestos a buscar algún curso en línea, una vez que ha iniciado su carrera profesional.

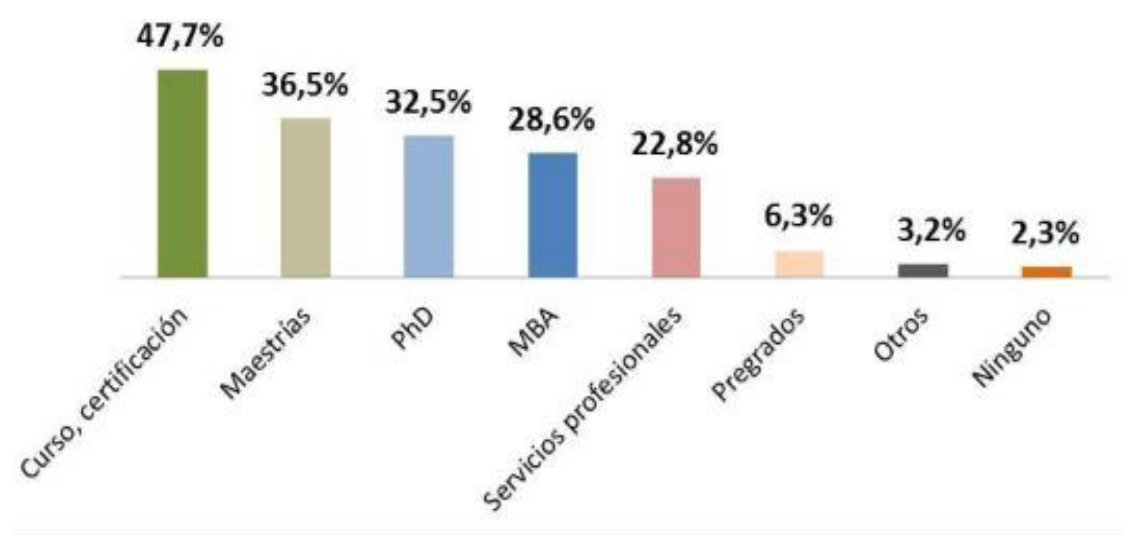

Figura 1. Encuesta sobre aceptación de cursos y medios online.

Fuente: World Economic Forum (2016)

José Manuel Santamans (2014) señala cuatro zonas en América Latina que se van a erigir como puntos de mayor crecimiento en educación virtual en los próximos 10 años. Brasil mostrará una expansión estimada en un $21,5 \%$, Colombia en un 18,6 \%, Bolivia en un 17,8 $\%$ y seguido de cerca por Chile en 14,4\%. En Brasil se pronostica que serán los centros educativos los principales consumidores de servicios y productos e-learning, mientras que en Argentina el proceso de crecimiento tendrá una gran importancia entre las empresas y corporaciones. Por su parte, serán los gobiernos nacionales, a través de sus diferentes planes, los principales motores de diseminación del aprendizaje on-line en países como Colombia, México y Ecuador. Según Fernández y Vallejo (2014) en Ecuador, "la educación virtual ha crecido muy lentamente; aunque se prevé un aumento significativo en los próximos años, el acceso a Internet sigue siendo muy deficiente" (p. 33), asegurando que las primeras universidades en formar parte de esta iniciativa fueron la Universidad Técnica Particular de Loja (UTPL), la Escuela Superior Politécnica del Ejército, la Universidad Católica Santiago de 
Guayaquil, la Escuela Politécnica Javeriana del Ecuador, la Universidad de Especialidades Espíritu Santo, la Universidad Tecnológica Equinoccial, entre otras. De igual forma, afirman que la educación en línea no debe ser vista como una opción secundaria o remedial de la educación presencial, es más solo debe ser diferenciada por los mecanismos o técnicas distintas utilizadas en los procesos de formación académica; sin embargo, la educación virtual es limitada debido a la baja cobertura de internet que existe en el país, por lo cual, a muchas personas les impiden ingresar a procesos de educación virtual.

Por otra parte, Rea y Medina (2016) afirman que "la educación superior a distancia virtual tiene algunos retos que superar en el Ecuador. El Estado debe fomentar políticas educacionales a la vanguardia de las necesidades de la sociedad" (p. 10). Si bien es cierto, las universidades ofertantes de esta modalidad de estudios tienen el deber de crear modelos pedagógicos que propicien la gestión del conocimiento, es decir, deberán trabajar el gobierno conjuntamente con las IES, para reducir las brechas socioeconomicas, se debe destacar que las TIC solas no cambiarán la educación; pero ayudan a generar nuevas formas de aprendizaje para los estudiantes en las universidades, lo cual permitirá desarrollar las competencias y habilidades de los profesionales acordes a las exigencias del mundo actual. De igual forma, Dávila y Ruiz (2016) señalan que "el e-Learning es una modalidad educativa que ofrece un gran potencial para la formación de calidad en el contexto de la sociedad de la informacion y del concimiento, sobre la base de docentes implicados en la ejecución de buenas prácticas en sus labores didácticas y tutoriales" (p. 18). Por lo tanto, resulta trascendental procurar tener una educación virtual de calidad, siendo su objetivo, lograr desarrollar un perfil de competencia adecuado para contar con profesionales idóneos en este mercado global altamente competitivo.

\section{MATERIALES Y MÉTODOS}

El presente estudio tuvo un carácter no experimental, de campo para tener una relación directa con los actores en su ambiente natural (Arias, 2016), transversal (Hernández, Fernández y Baptista, 2010) y descriptiva (Sabino, 2013). La muestra se definió entre una población de estudio conformada por un grupo de 150 estudiantes sin discriminar entre géneros ni rendimiento estudiantil, con edades comprendidas entre los 15 y 17 años, del último año de Educación Básica Superior de 5 colegios fiscales de la zona norte y sur de la ciudad de Guayaquil (ver Tabla 2) pertenecientes a los distritos que presentan mayores tasas de estudiantes que no logran obtener un cupo universitario de un Instituto de Educación Superior. 
Se diseñó un cuestionario estructurado de 10 preguntas, recolectando información empírica sobre las variables objeto de estudio con una escala de frecuencia tipo Likert de cuatro puntos (Muy de acuerdo, de acuerdo, en desacuerdo, totalmente en desacuerdo). Los datos fueron recolectados entre octubre y diciembre del 2018.

Tabla 2. Distribución de la muestra de estudio.

\begin{tabular}{|c|c|c|c|}
\hline Parroquia & Nombre del Distrito & Mujeres & Hombres \\
\hline Pascuales & Monte Sinaí & 16 & 15 \\
\hline Guayaquil & Monte Sinaí & 17 & 14 \\
\hline Tarqui & Pascuales 1 & 17 & 14 \\
\hline Tarqui & Tarqui 2 & 16 & 10 \\
\hline $\begin{array}{c}\text { Febres } \\
\text { Cordero }\end{array}$ & $\begin{array}{c}\text { Febres Cordero } \\
\text { Portete }\end{array}$ & 17 & 14 \\
\hline \multicolumn{2}{|c|}{ Total } & $\mathbf{8 3}$ & $\mathbf{6 7}$ \\
\hline
\end{tabular}

Fuente: Elaboración propia.

\section{ANÁLISIS DE LOS RESULTADOS}

La tabla 3 muestra la distribución de los indicadores de frecuencia, en donde el 30\% de los encuestados considera estar muy de acuerdo en tomar sus estudios universitarios de manera virtual sino alcanzan cupo en la universidad que desean, el $50 \%$ considera estar de acuerdo con esa opción, el $12 \%$ está en desacuerdo con realizar sus estudios de manera virtual y el $8 \%$ está totalmente en desacuerdo con esta forma de estudio y prefiere hacerlo de manera presencial.

En relación con la pregunta sobre si la educación virtual es mejor que la educación presencial se puede apreciar que un $5 \%$ de los encuestados dice estar muy de acuerdo que es mejor, un $12 \%$ está de acuerdo, un $63 \%$ está en desacuerdo y un $20 \%$ totalmente en desacuerdo. Entre los comentarios recibidos fueron que aún tienen muchas dudas acerca de lo que significa educación virtual. 

CPA.

Según lo consultado en el cuestionario solo $6 \%$ de los estudiantes encuestados dicen estar muy de acuerdo con el interés que le casusa los programas virtuales ofrecidos, el $22 \%$ dice estar de acuerdo, el $63 \%$ sigue sin que le cause interés alguno y el $9 \%$ está totalmente en desacuerdo con los programas ofrecidos de las carreras que han pensado seguir.

El 36\% de los estudiantes consultados dice estar en desacuerdo con asegurar que las universidades estén preparadas para dar clases de manera virtual, el 22\% dice estar totalmente en desacuerdo con que puedan, en especial las universidades públicas, el $29 \%$ dice estar de acuerdo con que si están preparadas y solo el $13 \%$ está muy de acuerdo en que puedan dar clases en esta modalidad virtual.

Con el fin de medir la capacidad de los estudiantes sobre la manera en que llevarían los estudios virtuales se les consultó si se les haría más difícil estudiar de manera virtual desde su casa que de manera presencial en la universidad y se puede observar en los resultados que el $45 \%$ dice estar muy de acuerdo en que les sería muy difícil, el 32\% dice estar de acuerdo, el $18 \%$ que está en desacuerdo y el $5 \%$ está totalmente en desacuerdo porque consideran que la educación recibida en su colegio les serviría mucho. 
Tabla 3. Síntesis de cuestionario a 150 estudiantes del último año de educación básica superior.

\begin{tabular}{|c|c|c|c|c|c|}
\hline $\begin{array}{l}\text { Porcentajes de Respuestas dadas } \\
\text { Pregunta realizada }\end{array}$ & $\begin{array}{l}\text { Muy de } \\
\text { acuerdo }\end{array}$ & $\begin{array}{l}\text { De } \\
\text { acuerdo }\end{array}$ & $\begin{array}{c}\text { En } \\
\text { desacuerdo }\end{array}$ & \begin{tabular}{|c|}
$\begin{array}{c}\text { Totalmente } \\
\text { en } \\
\text { desacuerdo }\end{array}$ \\
\end{tabular} & $\begin{array}{l}\text { Total de } \\
\text { la } \\
\text { muestra }\end{array}$ \\
\hline $\begin{array}{l}\text { ¿Usted consideraría seguir sus estudios } \\
\text { universitarios de manera virtual, en caso no de } \\
\text { alcanzar cupo de manera presencial? }\end{array}$ & $30 \%$ & $50 \%$ & $12 \%$ & $8 \%$ & $100 \%$ \\
\hline $\begin{array}{l}\text { ¿Usted considera que la educación virtual es } \\
\text { mejor que la presencial? }\end{array}$ & $5 \%$ & $12 \%$ & $63 \%$ & $20 \%$ & $100 \%$ \\
\hline $\begin{array}{l}\text { ¿La universidad a la que quiere aplicar tiene } \\
\text { programas académicos } 100 \% \text { virtuales que le } \\
\text { interesen seguir? }\end{array}$ & $6 \%$ & $22 \%$ & $63 \%$ & $9 \%$ & $100 \%$ \\
\hline $\begin{array}{l}\text { ¿Usted considera que en la actualidad las } \\
\text { universidades están preparadas para dar sus } \\
\text { clases de manera virtual? }\end{array}$ & $13 \%$ & $29 \%$ & $36 \%$ & $22 \%$ & $100 \%$ \\
\hline $\begin{array}{l}\text { ¿Considera que se le haría mas difícil estudiar } \\
\text { de manera virtual, en vez de hacerlo de manera } \\
\text { presencial? }\end{array}$ & $45 \%$ & $32 \%$ & $18 \%$ & $5 \%$ & $100 \%$ \\
\hline $\begin{array}{l}\text { ¿Considera que se le haría difícil estudiar de } \\
\text { manera virtual si no tuviera internet en casa? }\end{array}$ & $75 \%$ & $23 \%$ & $0 \%$ & $2 \%$ & $100 \%$ \\
\hline $\begin{array}{l}\text { ¿Considera que las plataformas virtuales son } \\
\text { una alternativa de estudios con excelencia? }\end{array}$ & $28 \%$ & $24 \%$ & $26 \%$ & $22 \%$ & $100 \%$ \\
\hline $\begin{array}{l}\text { ¿Considera que la educación virtual podría dar } \\
\text { mas oportunidades de escoger carreras a } \\
\text { estudiantes que no logran obtener cupo para } \\
\text { estudios presenciales en las Universidades } \\
\text { Publicas? }\end{array}$ & $23 \%$ & $33 \%$ & $21 \%$ & $23 \%$ & $100 \%$ \\
\hline $\begin{array}{l}\text { ¿En caso de que usted decida estudiar de } \\
\text { manera virtual, recomendaría a un amigo suyo } \\
\text { para que también escoja esta opción de } \\
\text { estudios? }\end{array}$ & $41 \%$ & $25 \%$ & $33 \%$ & $1 \%$ & $100 \%$ \\
\hline $\begin{array}{l}\text { ¿Considera que el estudio virtual le permitiría } \\
\text { combinar su trabajo y sus estudios de mejor } \\
\text { manera? }\end{array}$ & $55 \%$ & $20 \%$ & $25 \%$ & $0 \%$ & $100 \%$ \\
\hline
\end{tabular}

Fuente: Elaboración propia.

Asimismo, se les consultó si se les haría difícil estudiar de manera virtual si no tuvieran internet en su casa y el $75 \%$ respondió que está muy de acuerdo con que les sería muy difícil, el 23\% está de acuerdo debido a que no cuentan con los recursos para tener internet de manera permanente en su casa y el $2 \%$ respondió estar totalmente en desacuerdo; ya que ellos buscarían la casa de un familiar o amigo para que les preste el internet.

Además, se preguntó si consideran las plataformas virtuales como una alternativa de excelencia para sus estudios universitarios y el $28 \%$ considera estar muy de acuerdo con esta alternativa de estudios, el $24 \%$ está de acuerdo que la calidad es igual que la presencial, el $26 \%$ está en desacuerdo con que sea un estudio de excelencia y el $22 \%$ está en total desacuerdo con esta alternativa y preferirían sus estudios en forma presencial.

Con relación a la pregunta de considerar que la educación virtual podría dar más oportunidades de escoger carreras a estudiantes que no alcancen cupo para estudios presenciales tenemos que, el $23 \%$ considera estar muy de acuerdo, el $33 \%$ considera estar 
de acuerdo porque podrían estudiar, el $21 \%$ considera estar en desacuerdo debido a que prefieren esperar a escoger de manera presencial y el $23 \%$ está totalmente en desacuerdo en escoger dicha opción.

En cuanto a la pregunta de si recomendaría a un amigo que continúe sus estudios de manera virtual en la universidad tenemos que el $41 \%$ dice estar muy de acuerdo en hacerlo, el $25 \%$ está de acuerdo, el 33\% dice estar en desacuerdo debido a que no sabe cómo sería la educación y el $1 \%$ dice estar totalmente en desacuerdo en recomendar esta opción a un amigo.

Por último, dentro del cuestionario se les consultó si consideran que el estudio virtual les permitiría combinar el trabajo y el estudio de mejor manera y el 55\% está muy de acuerdo en que podrían manejar mejor su tiempo para combinar las dos actividades, el $20 \%$ está de acuerdo en que es una buena opción y el $25 \%$ está en desacuerdo con esta opción y prefiere dedicarse a una de las dos alternativas.

\section{DISCUSIÓN}

Se puede observar por la encuesta realizada que aún hay cierto desconocimiento acerca de lo que significa el estudio mediante una plataforma online por parte de la mayoría de las estudiantes, especialmente de las zonas rurales de la ciudad de Guayaquil, aunque lo perciben como una buena alternativa de estudios de no poder alcanzar cupos dentro de las clases presenciales. Según datos del Senescyt (2019) la Universidad Central del Ecuador (UCE), la Universidad de las Fuerzas Armadas, la Unemi, la Universidad Técnica de Manabí y la Universidad Técnica del Norte (UTN) son parte de una iniciativa estatal cuya oferta académica fue de 30.950 cupos para el primer proceso de nivelación, pero solo 13.652 personas se matricularon efectivamente; es decir, el $56 \%$ de la oferta no fue demandada. Seguramente el interés de preferir estudiar su carrera universitaria irá aumentando con el tiempo, ya que podrán acceder a esta educación desde sus hogares a través de las plataformas virtuales con el sello y la garantía que tiene todas las universidades de prestigio. Esta es una modalidad que se va adaptando a los cambios de la sociedad actual y que se caracteriza por la innovación y el acceso a mayores tecnologías.

Para generar confianza en los jóvenes las universidades deberán generar programas educativos en línea que sean óptimos y llamativos en su pensum educativo para los estudiantes que aún no se sienten seguros de escoger esta alternativa de estudios. Una vez que el prejuicio de que la educación online no es eficiente, seguramente muchos estudiantes van a preferir esta modalidad virtual que les permitirá manejar de mejor manera su tiempo. 
Tal como lo describe Fernández y Vallejo (2014) en Ecuador, la educación virtual ha crecido muy lentamente; aunque se prevé un aumento significativo en los próximos años, el acceso a Internet sigue siendo muy deficiente, lo que se ha podido en cierta manera confirmar con el estudio realizado de una muestra de estudiantes del último año de Educación Básica Superior de 5 colegios fiscales.

\section{CONCLUSIONES}

En relación con las preguntas consultadas a los estudiantes del último año de Educación Básica Superior de 5 colegios fiscales de la zona norte y sur de la ciudad de Guayaquil, se concluye que muchos de los escolares en contexto, perciben como adecuado o muy adecuado la alternativa del estudio en plataformas virtuales. No obstante, un grupo importante lo percibe todavía como no adecuado y una minoría sigue totalmente en desacuerdo con este tipo de modalidad, ya que aún tienen muy arraigada la tradición de las clases presenciales.

En función de las diferencias individuales que tienen estos dos métodos de estudio, se concluye que la gran mayoría de los escolares de las instituciones perciben como una buena alternativa el estudiar bajo plataformas virtuales en caso de no alcanzar el cupo dentro la universidad que desean, ya que seguirán siendo estudios con excelencia académica que ofrecen las universidades.

Cabe destacar que en el contexto objeto de estudio, los estudiantes perciben que este modelo de formación aplicado permite adquirir un fuerte compromiso con sus estudios, mucha preparación y dedicación, así como una fuerte disciplina para sacarlo adelante; y a su vez, les permitiría poder trabajar de ser el caso, ya que podrán manejar mejor su tiempo entre sus estudios y su trabajo, también podrán tomar en consideración los medios y plataformas que desee utilizar para acceder al contenido.

Aunque es una oportunidad para incrementar la oferta y permitir que más bachilleres lleguen a la universidad, aún queda mucho trabajo por realizar por parte de las Universidades en especial para los estudiantes de las zonas rurales, ya que son los que menos posibilidades tienen de entrar en las ofertas académicas presenciales por la falta de cupos existentes. 


\section{REFERENCIAS BIBLIOGRÁFICAS}

Álvarez, F., Rodríguez, J., Sanz, E. y Fernández, M. (2008). Aprender enseñando: Elaboración de materiales didácticos que facilitan el aprendizaje autónomo. Formación Universitaria, 6 (1), 19-28.

Arias, F. (2016). El Problema de Investigación: Introducción a la Metodología Científica. Caracas: Editorial Episteme.

Chen, H. et al. (2004). Integrated medical informatics with small group teaching in medical education. International Journal of Medical Informatics. 50(1-3), 59-68.

Dávila, A., y Ruíz, C. (2016). Propuesta de buenas prácticas de educación virtual en el contexto universitario. RED-Revista de Educación a Distancia, 1-21.

Fernández, K., y Vallejo, A. (2014). La educación en línea: una perspectiva basada en la experiencia de los países. Revista de Educación y Desarrollo, 29-39.

García, A. L., Ruíz, M. y Domínguez, D. (2007). De la educación a distancia a la educación virtual (1ra. Ed.), España: Ariel.

García, L. (2017). Educación a distancia y virtual: calidad, disrupción, aprendizajes adaptativo y móvil. Revista Iberoamericana de Educación a Distancia, 20(2), 09-25. doi: http://dx.doi.org/10.5944/ried.20.2.18737

Herman, A. (2009). Enseñar y aprender en la sociedad del conocimiento y de la información. Quito: Educación Virtual EPN.

Hernández, R., Fernández, C., y Baptista, P. (2010). Metodología de la Investigación (5ta ed.). México DF, México: Editorial Mc Graw Hill Interamericana S.A.

López, R. (2016). El aseguramiento de la calidad de la educación virtual. INEC, 207-226.

Gazzola, A., y Didriksson, A. (2016). Tendencias de la Educación Superior en América Latina y el Caribe. IESALC, 55-87.

Martínez, J. y Romero, A. (2017). Problemáticas relacionadas con la acreditación de la calidad de la educación superior en América Latina. Innovación Educativa, 79-96.

Matallana, O. y Torres, M. (2011). Caracterización de la educación superior a distancia en las universidades colombianas. Revista de investigaciones UñAD, 10, pp. 60-80. Recuperado de http ://datateca.unad.edu.co/contenidos/l 00103/100103_2013_2/Evaluacion_Nal/Articulo_3.pdf 
Noguera, I., y Gros, B. (2016). Mirando el futuro: Evolución de las tendencias tecnopedagógicas en Educación Superior. Revista Científica de Tecnología Educativa, 130-140.

Padilla, J., Vega, P., y Rincón, D. (2014). Tendencias y dificultades para el uso de las TIC en educación superior. Revista Entramado, 10(1), 272- 295.

Rendición de cuentas de cuentas de la Secretaria de Educación Superior, Ciencia, Tecnología e Innovación (2018). Recuperado de https://www.educacionsuperior.gob.ec/senescytces-y-ceaaces-presentaron-de-manera-conjunta-su-rendicion-de-cuentas-2017/

Sabino, C. (2013). El Proceso de la Investigación. Argentina: Editorial Lumen.

Sorlin, S. \& H. Vessuri (2007). Introduction: The Democratic Deficit of Knowledge Economies. Knowledge Society vs. Knowledge Economy: Knowledge; Power, and Politics, pp. 134.

Tichapondwa, M. \& Tau, D. (2009). Introducing distance education. Virtual University for the Small States of the Commonwealth. Recuperado de http://www.col.org/sitecollectiondocuments/introducing_distance_education.pdf

Unigarro, M. A. (2004). Educación virtual. (2da. Ed.), Colombia: UNAB. 